\title{
MODELING THE LIKELIHOOD FAILURE OF AGING UNDERGROUND PIPELINE
}

\author{
Abbas Zakaria Abdalla Mohammed \\ Chief Engineer of Mechanical Department \\ Atbara Station \\ Sudanese Pipelines Petroleum Company (SPPC) Sudan
}

\begin{abstract}
Pipeline is a valuable asset transporting very dangerous materials, so it has been safe and reliable, Tradition methods of maintenance are not enough. Applied failure predicting techniques is the important for environment and economy.

Modeling is constructed for the failure frequency of underground pipelines per kilometer year, as a function of pipe and environmental characteristics. The parameters in the model were quantified, with uncertainty, using historical data and structured expert judgment. Fifteen experts from institutes in the Netherlands, the United Kingdom, Italy, France, Germany, Belgium, Denmark and Canada participated in the study.
\end{abstract}

Keywords: Modeling, Underground pipeline, Corrosion, Third party interference, Environmental hazardous, Uncertainty analysis, Expert judgments, Risk analysis

\section{INTRODUCTION}

This research presents an overview of causes and frequency of failures of pipelines handling petroleum, the most significant causes are third party damage, corrosion, and mechanical failures (materials failure and construction defects). There are so many failure factors such as pipeline age, pipeline soil, diameter, location, material transported, and operation conditions, the data been collected used for calculating pipeline failure rates Fig 1. Reliable prediction of asset condition and its likelihood of failure (LOF) is one of the core requirements for a utility to establish effective asset management strategies for optimized maintenance, rehabilitation, and replacement plans. Although there have been many efforts to predict the failure of pipe asset, many utilities across, still find it challenging to effectively predict the likelihood of failure of our pipeline assets. still using subjective scales and rely on engineers' anecdotal experience and judgments. This study developed a holistic procedural framework that utilities can follow to develop a data driven LOF prediction model of their pipeline assets. The unique contribution of this paper is that the framework addresses issues that a utility will encounter from data collection and data organization to LOF prediction model development, and discusses possible solutions as well.

\author{
M.I. Shukri Professor \\ Department of Mechanical Engineering \\ Faculty of Engineering \& Technology, Nile Valley \\ University, Atbara, river Nile, Sudan
}

Historical performance records to demonstrate and validate the framework, the procedural framework developed in this research is anticipated to facilitate and accelerate the practical use of advanced data-driven methods for underground pipeline asset management, which will result in more reliable and high-quality.

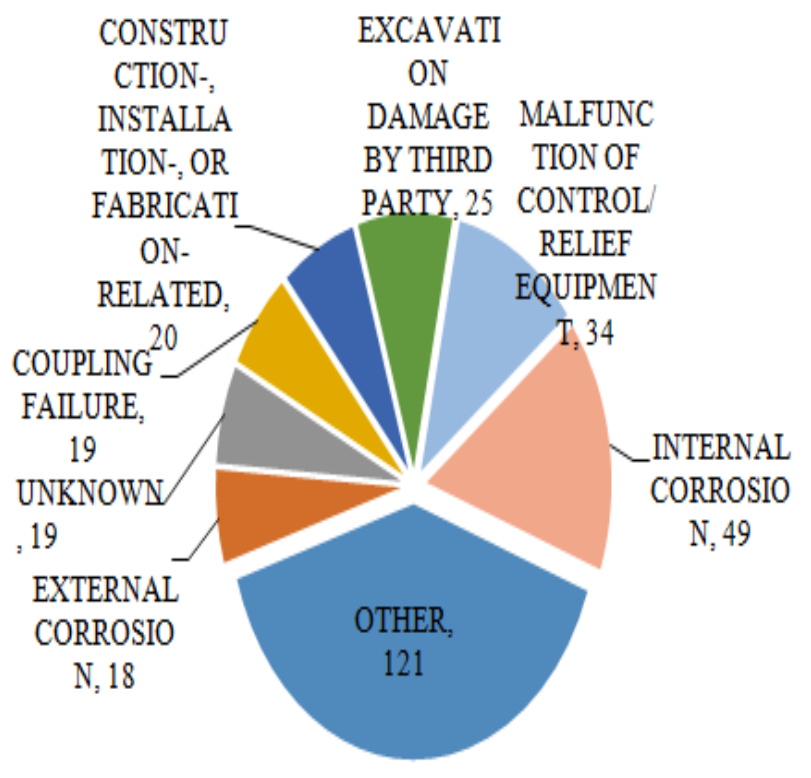

Fig. 1. Detailed representation of failure distribution of pipelines

\section{RESEARCH OBJECTIVES}

The research aims at developing a failure prediction model for oil pipelines, this model will allow oil pipeline operators to take those actions required to protect pipelines against the threats predicted and failure, to prioritize inspections. The following procedure was carried out to achieve this objective, also identify and study the most effective factors on the pipelines, age of failure and develop a model that predicts the age of failure to estimate the probability of failure of oil Pipelines. 


\section{International Journal of Engineering Applied Sciences and Technology, 2020 Vol. 5, Issue 8, ISSN No. 2455-2143, Pages 71-75 \\ Published Online December 2020 in IJEAST (http://www.ijeast.com)}

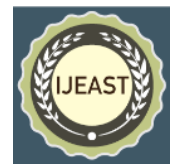

The study is to define the significant risk factors pertinent to the corrosion and excavation failures in the pipeline industry and subsequently check for reliability of the data in hand, also it may propose a model to predict the odds of survival of the pipeline. The data for the proposed model obtained from the Pipeline materials database of pipeline failure over the past of years. A statistical model to predict probability of pipeline failure in the forthcoming decade was developed by processing the data through the to get the significant parameters contributing to failure. The proposed model predicts that the chance of survival of the pipe, according to the data evaluated, decreases gradually with age for the of the pipeline life and remains within acceptable limits till its nominal expected life. the survival rate is almost constant and just about at the threshold level, the possible reasons contributing to this trend and a detailed analysis of the results based on the proposed model will be discussed in the research.

1 - This study developed a holistic procedural framework that can be followed to develop a data driven likelihood of failure prediction model of pipeline assets, and discusses possible solution.

2 - This study is anticipated to facilitate and accelerate the practical use of advanced data driven methods for underground pipeline asset management, which will result in more reliability.

3 - developing a generic framework for predicting pipe failure rate

Identify and study the critical factors of predicting the number of breaks and models to predict deterioration curves to predict the future condition of pipelines. Recognize the most sensitive factors to the number of breaks.

\section{PIPELINES FAILURE MODES}

Buried pipelines transporting oil is usually laid underground and exposed to varying boundary conditions. It has been often reported as industrial examples that many catastrophic disastrous pipeline accidents have been caused by the defect such as corrosion arisen by aging and environmental effects on pipelines. It is thus very important to investigate the effect of defect on the safety of buried pipelines for preventing heavy economical and social losses in advance. The buried pipelines usually have the various types of defects such as corrosion and environment assist cracking. If these were operated under an excessive operating pressure in the corroded pipelines, it could produce larger stresses than safety designed one. It may be emphasized that the failure analysis should be carried out with the help of the probability method than the conventional deterministic approach (CDA) because the (CDA) leads to uncertainties in the failure analysis with random variables imposed on varying boundary conditions

\section{FACTORS AFFECTING THE FAILURE RATE OF PIPELINES}

\section{Static and dynamic factor}

Stone et al. (2002) categorized factors contributing to the failure of water pipelines into two groups: static factors and dynamic factors. The characteristics of static parameters do not depend on the time, but dynamic factors' specifications change over time. Static parameters include the diameter, length, soil type, pipe material, etc. On the other hand, the age, cumulative number of breaks, soil corrosivity and water pressure are examples of dynamic factors influencing pipe failure rate. Osman and Bainbridge (2011) studied the effect of time-dependent variables like pipe age, temperature and soil moisture on the deterioration of water pipes. Static factors such as soil type, length, wall thickness and diameter of the pipe were not considered in their study because of the unavailability of reliable data.

\section{Physical, environmental and operational factors}

Infra Guide. (2003) classified the factors contributing to the deterioration of water pipes to three main categories; physical, environmental and operational as shown in. According to Infra Guide (2003), physical factors include pipe material, pipe wall thickness, pipe age, pipe vintage, pipe diameter, type of joints, thrust restraint, pipe lining and coating, dissimilar metals, pipe installation and pipe manufacture. In other researches, pipe length and buried depth are also known as physical factors Infra Guide (2003) considered pipe bedding, trench backfill, soil type, groundwater, climate, pipe location, disturbances, stray electrical currents, and seismic activity as the environmental, factors. While, other researchers included rainfall, traffic and loading, and trench backfill as the environmental factors as well. Kabir et al. (2015b) studied the effect of soil type on the failure rate of water pipelines and highlighted that soil type can be classified further to major and minor factors. The five major soil's factors include soil electrical resistivity, soil $\mathrm{pH}$, redox potential, soil sulfide contents and soil moisture. The five minor soil factors are; temperature of soil, oxygen contents, presence of acids, sulfates and sulfates reducing bacteria's. The Internal water pressure, transient pressure, leakage, water quality, flow velocity, backflow potential, and O\&M practices are examples of operational factors (Infra Guide 2003). Others considered the nature and date of last failure (e.g., type, cause, severity),

\section{RESEARCH METHODOLOGY}

This research is to identify the type of failure that menaces an oil pipeline by knowing some basic pipeline features, all of the failure modes threatening oil pipelines are studied as a perquisite to this identification process moreover, an extensive study of pipeline attributes and their influence on each type of failure cause is performed. The model's development is based 


\section{International Journal of Engineering Applied Sciences and Technology, 2020 \\ Vol. 5, Issue 8, ISSN No. 2455-2143, Pages 71-75 \\ Published Online December 2020 in IJEAST (http://www.ijeast.com)}

mainly on historical data presented in the publishes detailed Reports which displays all of the accidents that transpired in the pipeline system over the last years. The report cites the cause of each accident, which is the model's output, as well as some pipeline attributes, which are the model's inputs, methodology followed to achieve this goal consists of five main stages.

A literature review which presents the main causes of pipeline failures the

pipeline inspection techniques and a review of factors those contribute to pipeline failure.

i. Data collection and data preparation.

ii. Development of a failure prediction

iii. Development of a failure prediction model

iv. Sensitivity analysis and validation of the developed models

\section{FAILURE PREDICTION TECHNIQUES}

Researchers developed different models to predict the failure rate of pipes for a reliable infrastructure management. These failure prediction models are classified into four categories

\section{Deterministic Models}

Deterministic models usually are used in cases where the relationship between inputs and output is clear. In two approaches the deterministic models can be applied, empirical and mechanistic. Empirical approach tries to find the relation between failure rates as the output and the features and attributes of a group of pipes as the inputs. While, the mechanistic approach can forecast the remaining useful life of an individual asset (just one pipe). The problem of these models is that a deterministic model can be applied just in

\section{Statistical Models}

This type of modeling is typically used to predict the useful life or time to failure of infrastructure assets (Lawless 1983). Statistical models are applied to homogeneous groups of pipes or other infrastructure assets and need recorded failures or data regarding asset's condition. In this approach, regression is utilized to build a model based on the historical data that can predict the failure or condition of assets. In regression, the dependent variable is related to at least one of the independent variables.

\section{Probabilistic Models}

Probabilistic models analyze the probability of an event occurring (Creighton 1994). The probability of occurrence is one and the probability of the event that cannot happen is zero. The other probability of occurrence should be between 0 and 1 (Mitrani 1998). Information about asset conditions and attributes are required to develop a probabilistic model. The output or dependent variable would be a range of values instead of the specific number. These models need extensive data and typically used in infrastructure assets (Clair and Sinha 2012). It should be noted that the probabilistic approach commonly increases the computational complexity of the models (Moglia 2007)., many studies employed the probabilistic approach to develop water mains assessment models (Moglia et al. (2007), Li et al. (2009), Kabir et al. (2014), Jenkins et al. (2014), Francis et al. (2014), and Kabir et al. (2015b)). Moglia et al. (2007) developed a physical probabilistic failure prediction model based on the fracture mechanics of cast iron water pipes. The random independent variables were added to the inputs, and then Monte-Carlo simulation technique was applied to deal with the computational complexity of the model. The developed model without failure data, degradation and load data, was not capable of estimating failure rates of water pipes. Whereas, with these data, it can predict failure rates more accurately probability of buried pipes. They considered the effect of random inputs and used Monte-Carlo simulation framework to calculate cumulative distribution function (CDF) of remaining useful life of pipelines. But, they did not consider the correlation of defects for a pipeline having more than one corrosion defects. Also, they found CDF

\section{Artificial Intelligence Models}

In this literature review, Artificial intelligence models include Artificial Neural Networks and Fuzzy set theory models.

\section{i. Artificial Neural Networks}

Artificial Neural Network (ANN) is a method that can predict pipe failure and deterioration of infrastructure specially buried pipes. The ANN follows the pattern of the human brain using its generalization capabilities. Thus, this technique is able to process information even under large, complex, and uncertain environment. The high-quality database is needed for supervised training and forecasting the future condition of the pipes. Moreover, ANN needs several controlling factors including: number of hidden layers, the number of neurons in each hidden layer, activation functions, the number of training epochs, learning rate, and momentum term. However, ANN is considered as a "Black Box" technique. Therefore, it is not able to provide insight into the relationship between dependent and independents variables (Clair and Sinha 2012; Moselhi and Hegazy 1993, Atef et al. 2015, Shirzad et al. 2014).

\section{ii. Fuzzy Logic}

Fuzzy Logic is a mathematical method in the field of artificial intelligence that widely used by researchers to assign a value to a certain degree of membership instead of crisp values such as zero and one. This method is known to deal with systems that are subject to uncertainties and ambiguities. Fuzzy Logic is applicable in infrastructure assets like oil and gas, water, bridges and highways (Siler and Buckley 2005, Clair and Sinha 2012). Aydogdu and Firat (2014) incorporated two methods: fuzzy clustering and Least Squares Support 


\section{International Journal of Engineering Applied Sciences and Technology, 2020 \\ Vol. 5, Issue 8, ISSN No. 2455-2143, Pages 71-75 \\ Published Online December 2020 in IJEAST (http://www.ijeast.com)}

Vector Machine (LS-SVM) in order to estimate the failure rate of water pipes. At first, they developed failure rate estimation model using LS-SVM, and then fuzzy clustering method is utilized to define nine sub-regions for predictive performance improvement of the model. Afterward, the results were compared to the results of Feed

Forward Neural Network (FFNN) and Generalized Regression Neural Network (GRNN) methods. Finally, for model evaluation they employed some measurement indexes such as Correlation Coefficient (R), Efficiency (E) and Root Mean Square Error (RMSE)

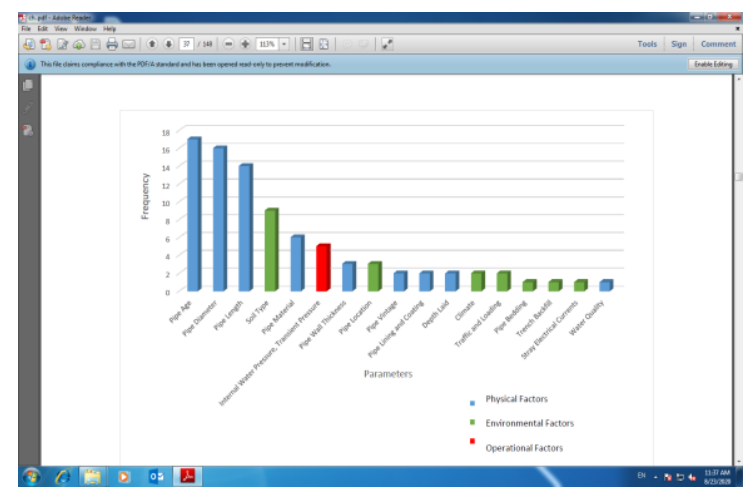

Fig. 2 Frequency of effective parameters

\section{CONCLUSION}

In this research, a probabilistic model consisting of an extreme value analysis and two corrosion rate models for uniform corrosion and pitting corrosion has been demonstrated to study internal corrosion of pipelines subject to the aqueous $\mathrm{CO}_{2} / \mathrm{H}_{2} \mathrm{~S}$ environment. This model is able to predict the corrosion rate based on specific corrosion types among uniform and pitting corrosion.

Gathering pipelines, were studied to validate the proposed model, probability distribution functions of operating and basic design variables were applied as input parameters for the proposed corrosion rate model for uniform corrosion and the model for pitting corrosion. Model predictions in terms of corrosion rate calculations were done by Monte Carlo simulation to consider the uncertainties of operating parameters. Most results show reasonable agreement between the observational corrosion rates and the model predictions in terms of median and mean values.

Older pipelines were found to be more vulnerable to pitting corrosion, whereas younger pipelines tend to suffer mainly uniform corrosion. It is suggested that older pipelines have a higher possibility of passive film breakdown due to the presence of chloride ions as well as surface tension effect at more accumulated flaws that make them vulnerable to pitting corrosion.

\section{REFERENCES}

(1) R.B. Petersen, R.E. Melchers, Corros. Prev. 23, (2012) Proposed probabilistic models of pipe failure in water distribution system ( pp 1-12)

(2) D.R. Kiran, in Total Quality Management, (2017) ) Failure Modes and Effects Analysis Modeling Deformation of Corroded Buried Steel Pipes and Design of Protective Measure: ( pp 1-7)

(3) Alawode A J and Ogunleye - (2011),"Maintenance, Security and Environmental Implications of Pipeline Damage and Ruptures in the Niger Delta Region", Pacific Journal of Science and Technology, Vol. 12, No. I, pp.( $515-573)$

(4) Irorukpo C (1998), "Environmental Impact Assessment and Human Concern in the Petroleum Industry: Nigeria's Experience", $9^{\text {th }}$ International Conference on the Petroleum Industry and the Nigeria Environment, Abuja, pp. (766-782).

(5) Osman, H., \& Bainbridge, K. (2010). Comparison of statistical deterioration models for water distribution networks. Journal of Performance of Constructed Facilities, ( pp 259266).

(6) Netto T A, Ferraz U S and Estfen S F (2005) "The Effect of Corrosion Defects on the Burst Pressure of Pipelines", Journal of Constructional Steel Research, Vol. 61, pp.( 1185-1204).

(7) Achim, D., Ghotb, F., \& McManus, K. (2007). Prediction of water pipe asset life using neural networks. Journal of Infrastructure Systems, (pp 26-30).

(8) W. K. Muhlbauer, , Burlington, MA, 2004. The Nigerian Oil and Gas Industy, Pipeline Risk Management Manual: Ideas, Techniques, and Resources, Gulf Professional Publishing and Elsevier (p.1-7),

(9) Onuaha F. C. (2008), "Oil Pipeline Sabbotage in Nigeria: Dimensions, Actors and Implications for National Security", African Security Review, Vol. 17,( p. 3).

(10) Pipeline Operators Forum (2009), Specifications and Requirements for Intelligent Pig Inspection of Pipelines. Version, p.( 38).

(11) Slesarev D and Sukhorukov D (2008), "Practice of In-Line Inspection withMDSCAN Intelligent 
Pigs", 17 th World Conference on Non-Destructive Testing. Shangai, China ( PP3-7\} .

(12) Castanier, B., \& Rausand, M. (2006). Maintenance optimization for subsea oil pipelines. International Journal of Pressure Vessels and Piping, (pp. 236-243 ).

(13) S. Nešić, "Key issues related to modelling of internal corrosion of oil and gas pipelines., (2007).- Areview," Corrosion science, vol. 49, pp. (4308-4338)
(14) C. De Waard and D. Milliams, (1975 ). "Carbonic acid corrosion of steel," Corrosion, (pp. 177-181 \},

(15) C. De Waard, U. Lotz, and D. Milliams, (1991 ). "Predictive model for $\mathrm{CO} 2$ corrosion engineering in wet natural gas pipelines," Corrosion, ( pp. 976-985) 